\title{
An automatic energy-saving and thermal monitoring/controlling system for a pond
}

\author{
Ching-Chien Cheng ${ }^{1}$, Min-Chie Chiu, ${ }^{2, *}$, Che-Min Chiu' ${ }^{3}$,Huang-Kuang Kung ${ }^{1}$,Chin-Yu Wang ${ }^{1}$, and Zi-Jie Gao ${ }^{2}$ \\ ${ }^{1}$ Institute of Mechatronics Engineering, Cheng Shiu University, Kaohsiung City, Taiwan 83347, R.O.C. \\ ${ }^{2}$ Department of Mechanical and Automation Engineering, Chung Chou University of Science and Technology, Taiwan, R.O.C. \\ ${ }^{3}$ Institute of Biomedical Engineering, National Tsing Hua University, Taiwan, R.O.C.
}

\begin{abstract}
Because of low temperatures and oxygen in cold water, fish will die when cold currents arrive. This will cause tremendous loss of money. In order reduce the cooling of the pond, an automatic thermal detecting and cold-roofing system using a wind-proofing device, heaters, and thermal detectors is proposed. To reduce heat loss due to thermal convection above the pond surface, a motor-driven wind-proofing device automatically controlled by a PLC controller is adopted. Here, the wind-proofing device, thermal detectors, and heating system are connected to the PLC controller. The PLC will also be connected to the PC interface. The temperature thresholds used to trigger the heater and the wind proofing device can be set at the PC interface. Two options for manipulating the heating and the automatic heating can be selected. The related wind-proofing area and the number of heaters will be determined according to the current temperature. Moreover, the PLC can be wirelessly connected to the server PC in the control room. The pond keeper can monitor everything online and control the pond water's temperature. With this, the problem of fish dying in a cold wave can be solved. Consequently, to reduce the electrical exhaust when heating up the pond water, green energy, solar energy and wind energy, is used.
\end{abstract}

\section{Introduction}

Primary factors of water quality affecting fish include adequate oxygen, proper temperature, transparency, and limited levels of metabolites. Earlier studies of the water quality of a fish pond in India were conducted by Sewell [1] and Pruthi [2]. During the winter months, plant growth and photosynthesis was limited by low water temperatures. Because of less photosynthesis, the reduction of oxygen caused problems for fish [3, 4]. In order to prevent fish from dying due to low oxygen and temperature when cold currents arrive, several strategies used in reducing the heat loss and increasing the temperature in water were needed and presented in the paper. To decrease heat loss during heat convection above the pond surface, an automatic wind-proofing device linked with a motor as well as a controller is adopted. To heat up the pond's water when the temperature is low, an online temperature monitoring system in conjunction with thermal sensors and heaters is established. Moreover, to save electrical power, green energy (wind and solar) installed around the pond is adopted.

Consequently, to facilitate the operation process, a PC-based interface connected to PLC is built. With this, the temperature thresholds used to trigger the heater and the wind proofing device can be set in the PC's interface.

\section{Automatic energy-saving, wind-proofing, and thermal monitoring/controlling system for a pond}

The structure of automatic energy-saving, wind-proofing, and a thermal monitoring/controlling system for a pond is depicted in Fig. 1. The related input/output diagram of the PLC connection is shown in Fig. 2. In order to remotely manipulate the energy-saving, wind-proofing, and thermal monitoring/controlling system via the server pc, the auxiliary relays defined in Table 1 have been added into the PLC's ladder program shown in Fig. 3. As indicated in Fig. 3, the auxiliary relays are marked in red in place of the buttons. The server pc can then submit the command to actuate the related functions for the energysaving, wind-proofing, and thermal monitoring/ controlling system. Moreover, the interface of the server PC programmed by the $\mathrm{VB}$ (Virsual Basic) is also illustrated in Fig. 4.

\footnotetext{
Corresponding author: minchie.chiu@msa.hinet.net
} 


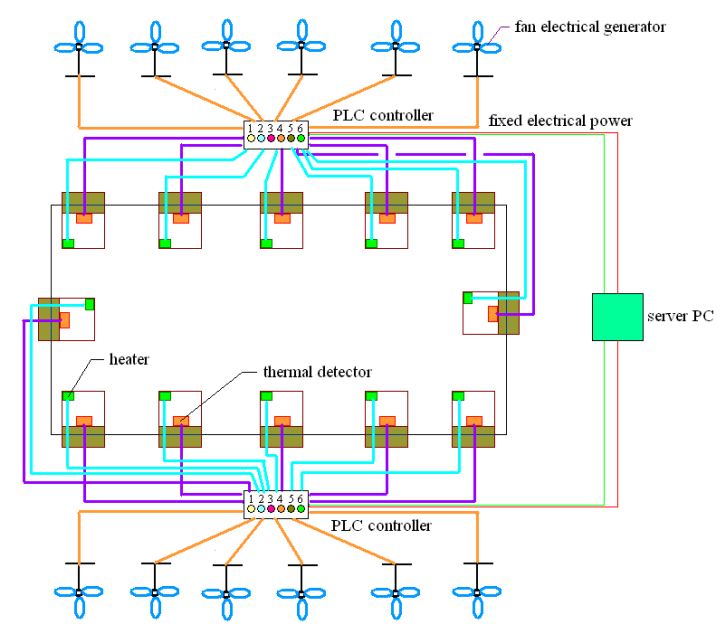

Fig. 1. The structure of automatic energy-saving, windproofing, and thermal monitoring/controlling system for a pond.

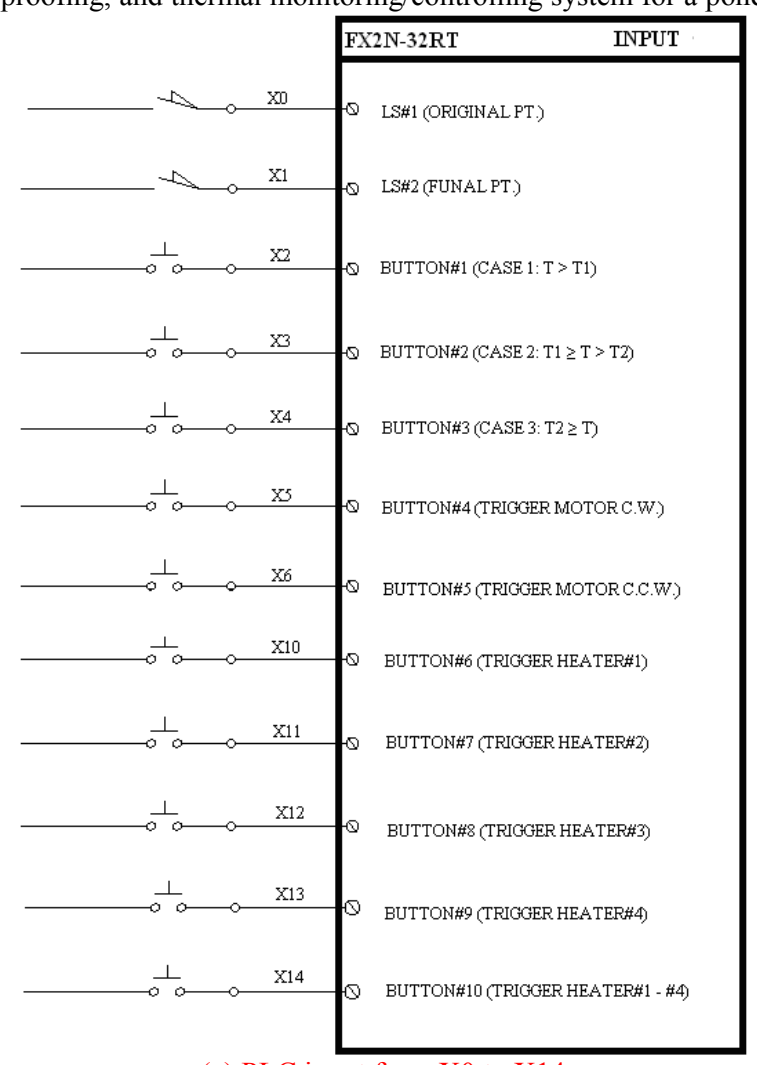

(a) PLC input from X0 to X14

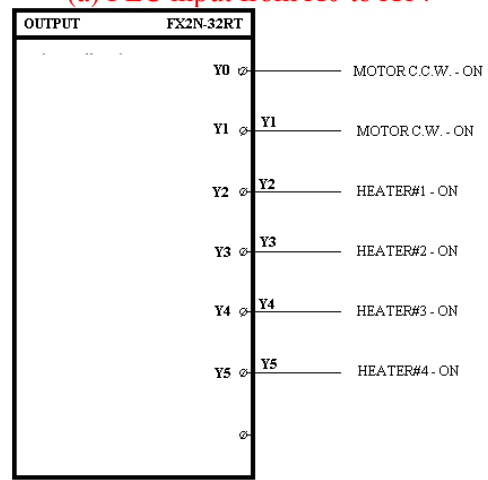

(b) PLC output from Y0 Y7 and Y10 Y12
Table 1. The auxiliary relays used to remotely control the action of the PLC program.

\begin{tabular}{|c|l|l|}
\hline $\begin{array}{c}\text { Auxiliary } \\
\text { Relay } \\
\text { Number }\end{array}$ & \multicolumn{1}{|c|}{ Action } & PLC Program \\
\hline M50 & $\begin{array}{l}\text { MOTOR C.C.W. } \\
\text { On/Off }\end{array}$ & SET, RST M50 \\
\hline M100 & $\begin{array}{l}\text { MOTOR C.W. } \\
\text { On/Off }\end{array}$ & SET, RST M100 \\
\hline M115 & $\begin{array}{l}\text { CASE 1 TESTING } \\
\text { On/Off }\end{array}$ & SET, RST M115 \\
\hline M116 & $\begin{array}{l}\text { CASE 2 TESTING } \\
\text { On/Off }\end{array}$ & SET, RST M116 \\
\hline M117 & $\begin{array}{l}\text { CASE 3 TESTING } \\
\text { On/Off }\end{array}$ & SET, RST M117 \\
\hline M201 & HEATER\#1 On/Off & SET, RST M201 \\
\hline M202 & HEATER\#2 On/Off & SET, RST M202 \\
\hline M203 & HEATER\#3 On/Off & SET, RST M203 \\
\hline M204 & HEATER\#4 On/Off & SET, RST M204 \\
\hline
\end{tabular}

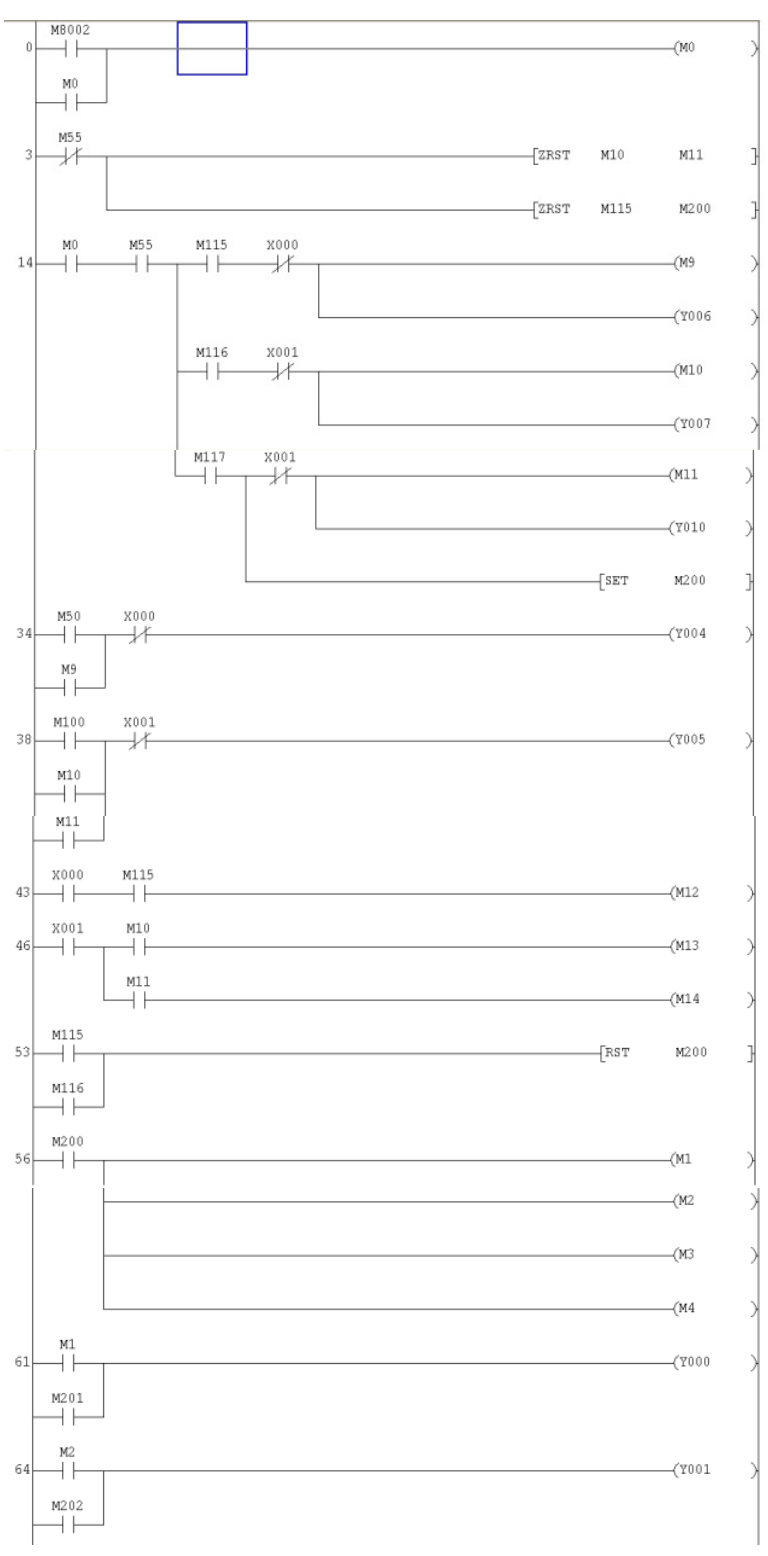

Fig. 2. The related wiring diagram of the PLC connection. 


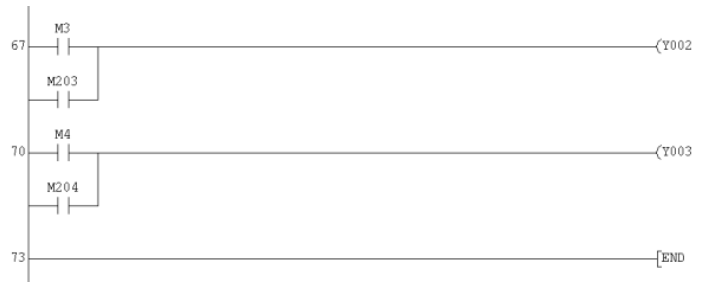

Fig. 3. The abstract of the related ladder program built into the PLC.

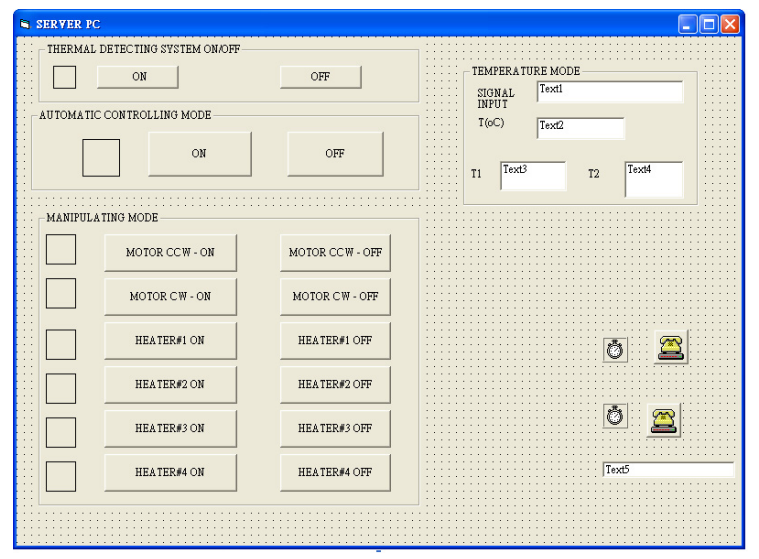

Fig. 4. The interface in the server PC.

\section{System established communication formated}

As indicated in Fig. 1 and Table 1, a PLC is used as a controller to actuate the actuators (one wind-proofing motor and four heaters) of the energy-saving, windproofing controlling mechanism. Also, a thermal detecting device installed inside a pond and connected to the server PC will feedback the thermal data to the server PC. The related wiring diagram of the system is shown in Fig.5. In addition, both the solar generator and the fan generator linked to the system are used to provide the necessary electrical power. In order to allocate the windproofing device, two limit switch sensors are installed at both ends of the pond. To increase the pond's water temperature, four sets of heaters are installed around the pond. Moreover, in order to remotely monitor/control the energy-saving, wind-proofing, and thermal monitoring/controlling system, wireless communication between the server pc and the PLC is established using the ZigBee module via the RS232/RS422 protocol. Thereafter, an interface at the server PC is programmed by the VB and is used to implement the system by sending a command to the PLC controller. The abstract of the related interface program built in the server $\mathrm{pc}$ is depicted in Fig. 6. To facilitate communication between the PLC and the PC via a RS232/RS422, the picket format for the MISTISUE FX2n-PLC's primary port communication is depicted in Table 2. Also, the definition of the picket format is shown in Table 3. The MISTISUE FX2n-PLC's extended port communication is shown in Table 4. In addition, the communication parameters used in the extended port are illustrated in Table 5. Consequently, the command description of the
MISTISUE FX2n-PLC's extended port communication protocol type 1 is shown in Table 6 .

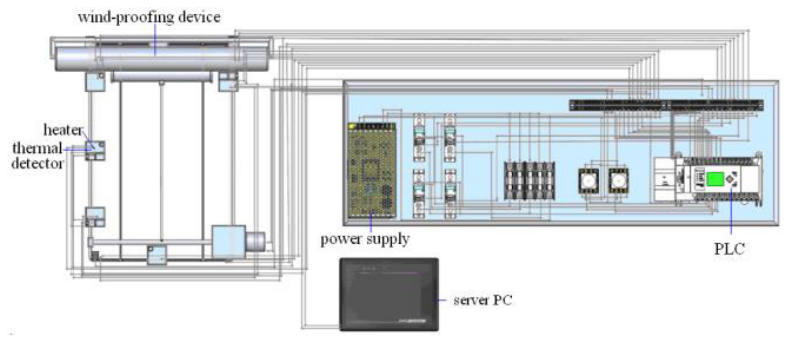

Fig. 5. The wiring diagram of the system.

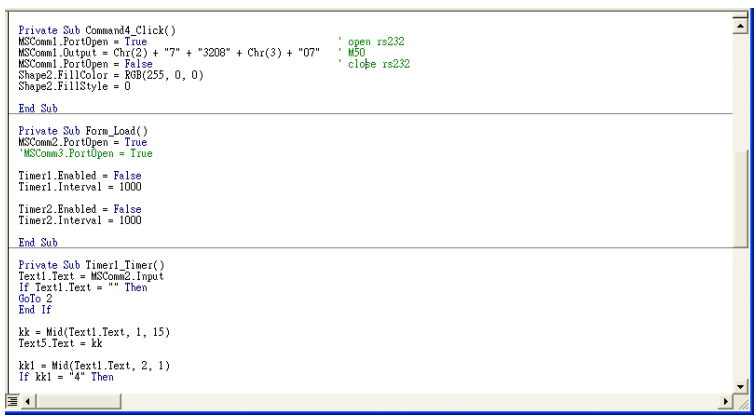

Fig. 6. The abstract of the related interface program built in the server PC.

Table 2. The communication packet format of the MISUBISHI FX2n-PLC's primary port.

\begin{tabular}{|c|c|c|c|c|}
\hline $\begin{array}{c}\text { Header } \\
\text { code }\end{array}$ & $\begin{array}{c}\text { Command } \\
\text { code }\end{array}$ & $\begin{array}{c}\text { Address } \\
\text { and data }\end{array}$ & $\begin{array}{c}\text { End } \\
\text { code }\end{array}$ & $\begin{array}{c}\text { Inspection } \\
\text { code }\end{array}$ \\
\hline STX (02) & CMD & DATA & $\begin{array}{c}\text { ETX } \\
(03)\end{array}$ & $\begin{array}{c}\text { Checksu } \\
\mathrm{m}\end{array}$ \\
\hline
\end{tabular}

Table 3. The definition of communication packet for MISUBISHI FX2n-PLC's primary port.

\begin{tabular}{|c|c|c|c|}
\hline $\begin{array}{c}\text { Command } \\
\text { category }\end{array}$ & $\begin{array}{c}\text { Command } \\
\text { code }\end{array}$ & Object & $\begin{array}{c}\text { Function } \\
\text { description }\end{array}$ \\
\hline Read & 0 & $\begin{array}{c}\mathrm{X}, \mathrm{Y}, \mathrm{M}, \mathrm{S} \\
, \mathrm{T}, \mathrm{C}, \mathrm{D}\end{array}$ & $\begin{array}{c}\text { Read the object } \\
\text { value }\end{array}$ \\
\hline Write & 1 & $\begin{array}{c}\mathrm{Y}, \mathrm{M}, \mathrm{S}, \mathrm{T}, \\
\mathrm{C}, \mathrm{D}\end{array}$ & $\begin{array}{c}\text { Write data to the } \\
\text { object }\end{array}$ \\
\hline Forcing on & 7 & $\begin{array}{c}\mathrm{Y}, \mathrm{M}, \mathrm{S}, \mathrm{T}, \\
\mathrm{C}\end{array}$ & $\begin{array}{c}\text { Forcing node to be } \\
\text { on }\end{array}$ \\
\hline $\begin{array}{c}\text { Forcing } \\
\text { off }\end{array}$ & 8 & $\begin{array}{c}\mathrm{Y}, \mathrm{M}, \mathrm{S}, \mathrm{T}, \\
\mathrm{C}\end{array}$ & $\begin{array}{c}\text { Forcing node to be } \\
\text { off }\end{array}$ \\
\hline
\end{tabular}

Table 4. The communication packet format of the MISUBISHI FX2n-PLC's extended port.

\begin{tabular}{|c|c|c|c|c|c|c|}
\hline $\begin{array}{c}\text { Header } \\
\text { code }\end{array}$ & $\begin{array}{c}\text { Station } \\
\text { number }\end{array}$ & $\begin{array}{c}\text { Computer } \\
\text { number }\end{array}$ & command & $\begin{array}{c}\text { Object } \\
\text { number/ } \\
\text { data }\end{array}$ & $\begin{array}{c}\text { End } \\
\text { code }\end{array}$ & $\begin{array}{c}\text { Insp- } \\
\text { cotion } \\
\text { code }\end{array}$ \\
\hline
\end{tabular}

Table 5. The parameter of the extended port's communications protocol for the MISUBISHI FX2n-PLC.

\begin{tabular}{|c|c|c|}
\hline D8120Bit & Name & Content \\
\hline 15 & Protocol type & $\begin{array}{c}0: \text { type 1, 1: type } \\
4\end{array}$ \\
\hline 14 & protocol & $0:$ No, $1:$ Yes \\
\hline 13 & Inspection code & $\begin{array}{c}0: \text { manipulate, } 1: \\
\text { auto }\end{array}$ \\
\hline 12 & Type of control line(II) & none \\
\hline 11 & DTR inspection mode & none \\
\hline
\end{tabular}




\begin{tabular}{|c|c|c|}
\hline 10 & Type of control line(I) & none \\
\hline 9 & End code & none \\
\hline 8 & Header code & none \\
\hline 7 & Transmission speed & $0011: 300$ \\
\cline { 1 - 1 } & & $0222: 4800$ \\
\cline { 1 - 1 } 5 & & $0100: 600$ \\
\cline { 1 - 1 } & & $1000: 9600$ \\
& & $0101: 1200$ \\
& & $1001: 19200$ \\
& & $0110: 2400$ \\
\hline 3 & Stop bit & $0: 1$ bit, $1: 2$ bits \\
\hline 2 & Parity check & $00:$ none; $02:$ \\
\cline { 1 - 1 } & & even; $01:$ odd \\
\hline 0 & Data bit & $0: 7$ bits $; 1: 8$ bits \\
\hline
\end{tabular}

Table 6. The command of the extended port's communications protocol for the MISUBISHI FX2n-PLC

\begin{tabular}{|c|c|c|}
\hline Command & Description & Available object \\
\hline BR & Read PLC's bit data & X, Y, M, S \\
\hline WR & Read PLC's bit_set data & D, TN, CN \\
\hline BW & Write data to PLC's bit & Y, M, S \\
\hline WW & $\begin{array}{c}\text { Write data to PLC's } \\
\text { bit_set }\end{array}$ & D, TN, CN \\
\hline BT & $\begin{array}{c}\text { Hybrid type of the } \\
\text { writing for bit }\end{array}$ & Y, M, S \\
\hline WT & $\begin{array}{c}\text { Hybrid type of the } \\
\text { writing for bit_set }\end{array}$ & D, TN, CN \\
\hline RR & Actuate PLC's running & \\
\hline RS & Actuate PLC's stop & \\
\hline PC & $\begin{array}{c}\text { Read the PLC's } \\
\text { category }\end{array}$ & \\
\hline
\end{tabular}

\section{The program design for serial communication between the server PC and the client PC}

The TCP/IP communication between the server pc and the client $\mathrm{PC}$ is performed using the WINSOCK object [5-9]. The host (server PC) can also be connected simultaneously by multiple client's PCs. The interface of the client $\mathrm{pc}$ is depicted in Fig. 7. Also, the related communication diagram is shown in Fig. 8. The communication process for the server port and the client port is described below.

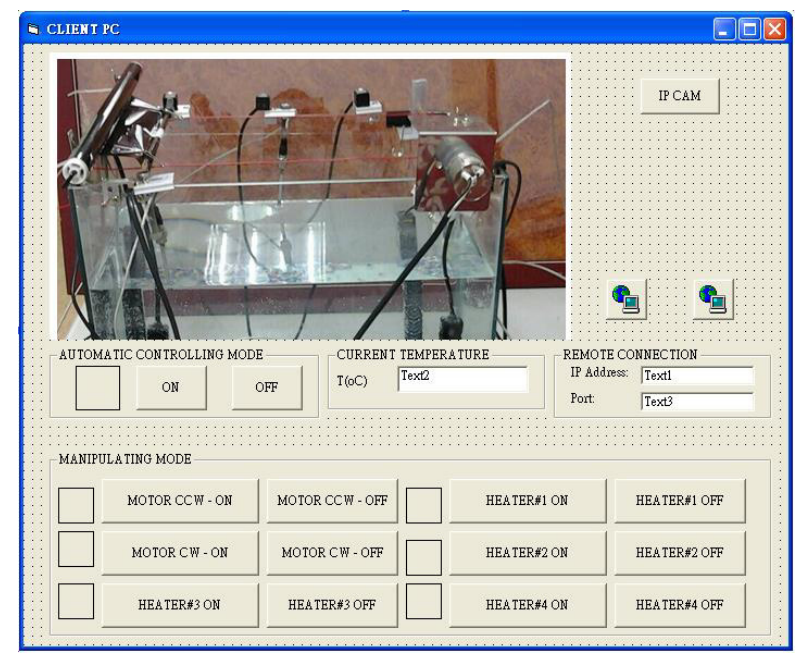

Fig. 7. The interface in the client PC.

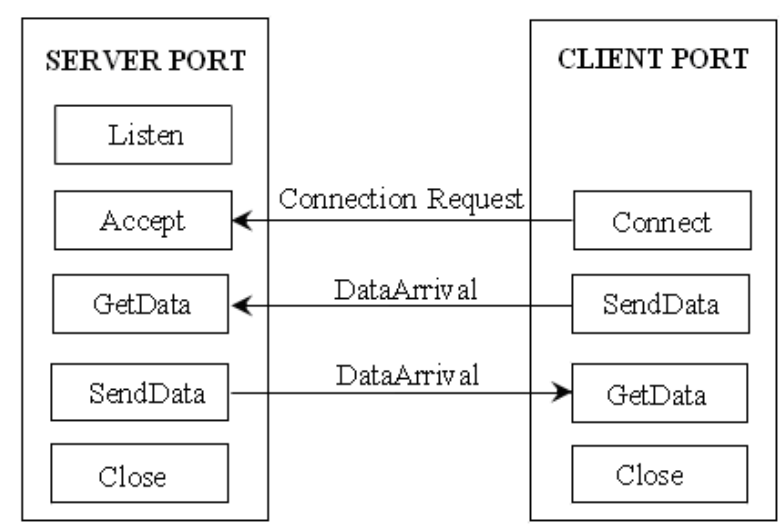

Fig. 8. The communication process between the client port and the server port.

\subsection{The communication process (server PC viewpoint) [8]:}

\section{(1) Listen:}

Because the server port will build a connection platform for the client port, a condition of "Listen" for the server $\mathrm{pc}$ is needed. The server port waits for the connection from the client port when "Listen" is ready. Moreover, because a WINSOCK object in the server port can be adopted for providing a connection for only one client, multiple WINSOCKs used in the program are requisite for a network of multiple clients connected to the server port. Hence, multiple WINSOCKs will be created using the "Load" command. One of the WINSOCKs (Winsock1(0)) is responsible for the "Listen" function. A "Local Port" will be stipulated as the port of "Listen."

(2) Accept:

The server port will receive an event ConnectionRequest from the client port when the client port submits a "Connect" for a further internet connection. The server port also accepts the client port's connection request using "Accept" via another WINSOCK.

(3) GetData:

The server port receives an event "DataArrival" from the client port when the client port sends data back to the server port. The server port will also receive data by using "GetData."

(4) SendData:

The server port sends data to the client port using "SendData."

\subsection{The communication process (client PC viewpoint) [10]:}

(1) Connect:

The WINSOCK in the client pc submits a connection request using "Connect." At this time, the WINSOCK will assign the server port's "Port Number" (at "Listen") to be the value of its "Remote Port."

(2) SendData:

The client port sends the data set to the server port using "SendData" when the internet connection between the server port and the client port is built.

(3) GetData: 
The client port will generate an event "DataArrival" when the server port sends a data set to the client port. The client port can receive the data using "GetData." (4) Close:

The end connection using "Close" is needed when the communication is finished.

\section{Results and Discussion}

\subsection{Results}

As indicated in Fig. 1, a prototype of a pc-base energysaving, wind-proofing, and thermal monitoring/ controlling system is established and shown in Fig. 9. A PLC is used as a controller to control the various actuators, including one motor and four heaters.

Here, the motor will be triggered by the PLC to forward or retrieve the wind proofing device when the current pond's water temperature detected by the thermal sensors reaches two thermal thresholds of pond water. A thermal sensor is adopted online to detect the temperature of the pond water. Two limit switches are installed at the two ends of the pond. A wireless communication between the server PC and the system is constructed using the ZigBee module connected to the PLC via the RS232/RS422 protocol. As indicated in Fig. 4 , an interface at the server $\mathrm{PC}$ is established to implement the system by sending a command to the PLC controller. Moreover, to realize the current temperature of the pond water, a remote distributing, monitoring, and controlling network in conjunction with an IP CAM via the TCP/IP network is built and shown in Fig. 7. To assure the operation of energy-saving, wind-proofing, and the thermal monitoring/controlling system, a visual monitoring system using the IP CAM is also adopted.

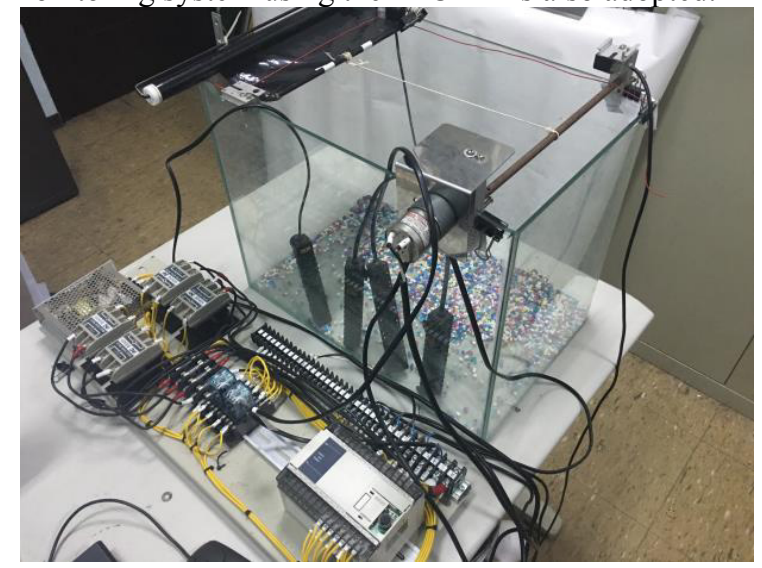

Fig. 9. A prototype of the automatic energy-saving, windproofing, and thermal monitoring/controlling system for a pond .

\subsection{Discussion}

The main issues concerning a pc-base energy-saving, wind-proofing, and thermal monitoring/controlling system follow :
(1) An electricity supplement extracted from solar energy and wind energy.

(2) Automation for keeping the pond water at an appropriate temperature and saving manpower.

(3) Establishing the client-server control system between the server PC and the client PC.

(4) Saving manpower on site by using an interface between the server PC and the system.

(5) Establishing the system's hardware and setting up a communication format between the PLC, ZigBee, and the server PC.

(6) Assuring the operation of energy-saving, windproofing, and the thermal monitoring/controlling system using an IP CAM visual monitoring system.

\section{Conclusion}

It has been shown that to reduce manpower and to maintain an appropriate temperature in the pond, a remote energy-saving, wind-proofing, and thermal monitoring/controlling system is necessary. This network is utilized using an inquiry between the server $\mathrm{PC}$ and the client PC via the TCP/IP network. To avoid interference from internet wiring with existing equipment, a wireless network for the system using the ZigBee module is adopted.

Consequently, to assure the operation of controlling temperature in pond water when cold currents arrive, an IP CAM visual monitoring system is established.

\section{References}

1. R. B. S. Sewell, J. of the Asiatic Society of Bengal, 22, 177 (1927).

2. H. S. Pruthi, Review of Hydrobiology Hydrographic, 26, 242 (1932)

3. Rossana Sallenave, New Mexico State University, Guide W-105, 1 (2013)

4. T. Zhu, C. Ringler, International Food policy Research Institute, 00961 (2010)

5. H. C. Cheng, M. C. Chiu, C. L. Huang, P. C. Chuo, I. Tech. J., 11(6), 686 (2012)

6. H. C. Cheng, M. C. Chiu, App. Mech. and Mat., 336-338, 1211 (2013)

7. H. C. Cheng, M. C. Chiu, J. I. O. S., 34(6), 373 (2013)

8. M. C. Chiu, H. C. Cheng, J. Inter. Math., 18(5), 493 (2015)

9. H. C. Cheng, M. C. Chiu, C. M. Chiu, C. Y. Yang, Sylwan Journal, 160(11), 147 (2016)

10. H. C. Cheng, M. C. Chiu, I. J. on Smart Sens. and Intell. Syst., 7(3), 1378 (2014) 